\section{"CARE" in social media: perceptions of reputation in the healthcare sector}

\author{
Vidhi Chaudhri, Tessa Oomen, Jason Pridmore and Alexandra Joon \\ Media and Communication, Erasmus University Rotterdam, \\ Rotterdam, The Netherlands
}

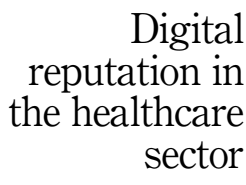

Received 22 June 2020 Revised 26 September 2020 Accepted 6 November 2020

\begin{abstract}
Purpose - Guided by the growing importance of social-mediated organisational communication, this study examines how communication professionals within healthcare organisations perceive and respond to the reputation impacts of social media on the organisation's reputation. Although the healthcare sector finds itself in the midst of a (continually) transforming landscape characterised by large amounts of digital health (mis) information and an empowered "patient-as-consumer", little is known about how professionals in this sector understand the changes and respond to them. Moreover, much extant scholarship on the topic is published in specialised health or medical journals and does not explicitly address the communication implications for healthcare organisations.

Design/methodology/approach - In-depth semi-structured interviews were conducted with communication professionals responsible for social media across eight hospitals in the Netherlands. The sample included two participants working as communication consultants/social media advisors for healthcare organisations. In all, 15 interviews were conducted.

Findings - Building on interviewee perspectives, the authors advance the CARE (Control, Access(ability), Responsive(ness) and Engagement) model of social-mediated communication, highlighting the dualistic characteristics of each dimension. This model is built upon a careful analysis of healthcare professional responses. In an always-on environment, understanding and managing the tensions within the authors' model may be decisive to the reputation implications of social media use.

Originality/value - Understanding the tensions within each dimension lends a more nuanced perspective on the potential impact(s) of social media as experienced by professionals in the field. In shifting away from a binary, either/or approach, the paper contributes to explicating the complexities of a pervasive phenomenon (i.e. social-mediated communication) and its multifaceted impacts on the healthcare sector.
\end{abstract}

Keywords Organisational communication, Healthcare, Netherlands, Paradox, Reputation, Social media

Paper type Research paper

\section{Introduction}

On Saturday, 29 January 2017, an 86-year-old man was injured in a car accident in Rotterdam, the Netherlands, and was taken to hospital (de Koning, 2017). Unfortunately, several mistakes were made in treating the man's injuries and his son complained about his treatment on social media. He indicated that he was prepared to sue the hospital for its negligence. This message was picked up by the newspaper $A D$, which forced the hospital to order an investigation into the incident. In another case, a University student sued Northwestern Memorial Hospital when a doctor took pictures of her receiving treatment for "extreme intoxication" and posted them on Facebook and Instagram (CBS, 2013). The student filed a lawsuit seeking US\$1.5

Vidhi Chaudhri, Tessa Oomen, Jason Pridmore and Alexandra Joon. Published by Emerald Publishing Limited. This article is published under the Creative Commons Attribution (CC BY 4.0) licence. Anyone may reproduce, distribute, translate and create derivative works of this article (for both commercial and non-commercial purposes), subject to full attribution to the original publication and authors. The full terms of this licence may be seen at http://creativecommons.org/licences/by/4.0/legalcode

The authors thank the editor and two anonymous reviewers for their constructive feedback and recommendations. A version of this paper was presented at the 10th Asian Conference on Media, Communication and Film (MediAsia) in October 2019.

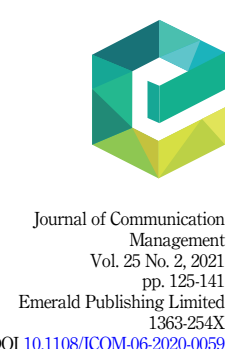

DOI 10.1108/JCOM-06-2020-0059 
$\mathrm{JCOM}$

25,2

million in damages in anticipation of the negative impact on her career prospects. In a wider critique of medical negligence and maltreatment, hashtags such as \#DoctorsAreDickheads and \#MyDoctorSaid signal a growing outpouring of patient complaints on social media (Vogel, 2019).

Such incidents are increasingly becoming more common in large part due to the rapid digitalisation of communication, especially via social media, which now also permeates the healthcare context. A report found that social media had fuelled a rise in complaints against doctors in the United Kingdom, nearly doubling from 5,168 in 2007 to 10,347 in 2012 (BBC, 2014). Whether the complaints reflected a deterioration of medical standards was unclear; however, two pertinent observations were made: a changing patient profile that is more aware and has higher expectations of their doctors, and the role of negative press on social media that could progressively "chip away" at the medical profession's reputation (BBC, 2014).

It is undeniable that in the last few years, the Internet and, in particular, social media have had a major impact on every industry. Social media are defined as "a group of Internet-based applications that build on the ideological and technological foundations of Web 2.0 and allow the creation and exchange of user generated content" (Kaplan and Haenlein, 2010, p. 61). Participation on social media by companies and organisations has been historically problematic when there was not a clear sense of strategic engagement for these organisations (see for instance, Fornier and Avery, 2011), highlighting a critical dimension (i.e. the lack of control) that is the centre point of conversations about the perceived impacts of social media.

The healthcare sector is not immune to these communication shifts. Building on the affordances of web 2.0, the concept of Health 2.0 is proposed as a participatory system of healthcare characterised by "active participation, with direct communication between patients, between professionals, and between patients and professionals" (RVZ, 2010). Fuelled by social media, developments in which "the patient is no longer a passive observer but an active participant in the healthcare process" (RVZ, 2010) have far-reaching implications which need to be examined in-depth.

As a result, there is a burgeoning body of research investigating the effects of social media on healthcare. However, much extant scholarship on the topic concerns itself with patients' or healthcare professionals' use of social media and/or the e-health communication between patient and provider. Few articles, if any, focus on the implications of social media for the reputation of healthcare organisations. Our study aims to fill this gap and examines how communication professionals within Dutch healthcare perceive and respond to the use of social media in their organisations. As a result of our analysis, we introduce a model of social media use that highlights the tensions and complexities that communication professionals must navigate as they seek to leverage social media for their respective organisations. Labelled CARE-Control, Access(ability), Responsive(ness) and Engagement - the model lends a more nuanced perspective on the potential impact(s) of social media as experienced by professionals in the field. This is a timely endeavour because in spite of the organisational uptake of social media, digitalisation-led challenges (e.g. speed and volume of information, social media management, matching audiences and channels and big data) are successively named as the top three issues confronting communication professionals in Europe (European Communication Monitor, 2019), issues for which healthcare organisations are not exempt.

\section{Social media in healthcare organisations}

As mentioned, research on social media use in the healthcare sector is on the rise; however, with some exception (e.g. Lim, 2016; McCarroll et al., 2014), much of this scholarship is published in specialised health or medical journals (e.g. Journal of Medical Internet Research; Telemedicine and e-Health) and does not explicitly address the reputation implications for 
healthcare organisations. For example, a longitudinal study of social media in 12 Western European hospitals reported a growing usage trend across the board (van de Belt et al., 2012). Notably, the Netherlands and the United Kingdom reported the highest use of social media in the study while Facebook was the dominant network with a presence in all countries.

Beyond the numbers, some research examined how hospitals are using social media. A study of US hospitals (Richter et al., 2014) found that although seven in ten hospitals used Facebook, the majority relied on an information dissemination strategy (e.g. patient education, announcing hospital and staff awards) rather than an engagement orientation (less than one-third). Similar results are echoed in Jiang (2019) research of "functional interactivity" features in Chinese hospitals' Sina Weibo (Chinese equivalent of Twitter) accounts whereby organisational disclosure and information dissemination were significantly more prominent than audience involvement.

Relative to the types of social networks and usage practices, less is known about why the healthcare sector is gravitating toward social media; in other words, understanding why social media are relevant to healthcare (Bermúdez-Tamayo et al., 2013). Although the ubiquity of social media make this question seem redundant, it is important to have a clear purpose for social media use and be cognisant of the potential benefits and risks. As Keller et al. (2014) contend, "public discourse on [. . . ] critical health topics continues to be influenced and sometimes shaped by discussions online from Twitter to Facebook", making it imperative for health professionals and organisations to make well-informed decisions about whether or not to be present in the social media space.

The transformational effect of digital technologies and innovations on healthcare services is unmistakable. Key among these will be the adoption of cloud services to improve data sharing between healthcare providers and increase computing capabilities associated with data processing (Report forthcoming). Another important development is the growth of virtual or telehealth solutions, which is driven by "informed and empowered consumers" who seek convenient and accessible information and are no longer passive targets for "push" solutions from business (Deloitte, 2019). Therefore, an examination of new communication technologies and their impacts on the healthcare sector is not only timely but may also facilitate a better understanding of the conditions that facilitate and constrain professional practices (e.g. Lim, 2016).

The importance of accessibility and transparency of health information is reinforced by Cordina and Greenberg (2020). Their survey-based study confirms the growing use of varied online sources (31\% hospital or physician website; $37 \%$ social media or apps; $20 \%$ health insurer's website or app) to make healthcare decisions from choosing a primary care provider or pharmacy to assessing quality of a physician or facility. Crucially, $68 \%$ of survey respondents consider facility and provider reputation in deciding where to receive care, and negative online reviews are decisive to patient experience ( $45 \%$ of respondents reported looking for a different provider after reading such reviews). These findings mirror a shift toward participatory medicine (Health 2.0) that allows "physicians and patients to digitally connect and take an active role in managing health" (Lim, 2016, p. 966). This is connected to and seen to give unbridled access, via "the patient's voice" (Lim, 2016, p. 967), to peer feedback and experiences with healthcare providers that could potentially be input for improving the quality of care. From a strategic standpoint, Kotsenas et al. (2018) note that a digital presence can contribute to building the (organisation and/or individual) brand, expanding the organisation's reach and generating business (e.g. through word-of-mouth referrals). From a consumer perspective, having access to transparent (online) information affords them the possibility to make better healthcare decisions accounting for the provider's reputation, quality and cost of care (Cordina and Greenberg, 2020).

The Netherlands is no exception to the transforming "patient as consumer" profile. Depending on their insurance, patients are free to make decisions on where to go for care 
$\mathrm{JCOM}$

25,2

(Vuijst, 2009). Research by the Nederlandse Vereniging van Ziekenhuizen [The Dutch Association of Hospitals] found that $25 \%$ of the patients will not automatically choose the closest hospital and between 65 and $90 \%$ prefer to opt for a specialised hospital (NVZ, 2011). Moreover, rankings of healthcare service providers are being published by newspapers and agencies based on preselected quality indicators and patient surveys. In 2013, the Internet had already become the main source for patients to find health-related information as well as way for patients to connect and communicate with healthcare providers. By then, nearly a third of the Dutch population used the Internet to find ratings of healthcare providers online (van de Belt et al., 2013). On ZorgkaartNederland.nl, "the largest experience site for Dutch healthcare where people share their experiences with healthcare with each other" [translated from Dutch], there are nearly 700,000 ratings of healthcare providers. In addition, owing to the vast accumulation of health information online, patients have become more aware and critical of the possibilities offered by health professionals, thereby challenging the assumption of "the doctor knows best" and thus pressuring hospitals to adapt to this new market of empowered patient-consumer (e.g. Waeraas and Maor, 2015).

Clearly, the pervasiveness of social-mediated communication has its pros and cons, for example, the potential for misinformation, violation of (patient) privacy, and risk to professional image and reputation (Lim, 2016; Ventola, 2014). In light of the aforementioned digitalisation-led changes, it becomes imperative to examine how communication professionals in the healthcare sector address the impacts of these developments with a specific focus on organisational reputation.

The (digital) reputation imperative and its implications for the healthcare sector A classic definition of reputation deems it "a perceptual representation of a company's past actions and future prospects that describe the firm's overall appeal to all of its key constituents when compared to other leading rivals" (Fombrun, 1996, p. 36). In other words, reputation refers to "the perceptions by stakeholders of an organisation's ability to fulfil their expectations" (Fombrun and van Riel, 2007, p. 43). Reputations are a firm's most valuable intangible assets and likened to a "magnet" for stakeholder support, access to resources, alignment with strategic objectives, competitive differentiation, stakeholder support, trust and pride in an organisation and even a "buffer" in times of crisis (Fombrun and van Riel, 2007).

From a communication standpoint, reputations are fluid and dynamic, constituted through ongoing interactions between organisations and their stakeholders or among groups of stakeholders independent of the organisation (Ravasi et al., 2018). Therefore, fully controlling or even managing reputation is an elusive goal (Aula and Heinonen, 2016), although organisations may, to some extent, influence reputation by how they behave, communicate and perform (Zarkada and Polydorou, 2014). The exponential growth of social media exacerbates the tension between control and reputation management as it changes how stakeholder evaluations are formed and disseminated in the public sphere. Etter et al. (2019) contend that new forms of uncontrolled interaction accompanied by real-time reach and a fragmented media environment challenge the homogeneity of evaluations that stakeholders make of organisations. Among the most significant developments, these authors highlight the shifts in communication flows (top-down to horizontal networks), audience roles (senders, receivers and co-producers with enhanced agency) and nature of content (emotional as opposed to "informational"; lower regard for "balance and accuracy") as fundamentally transforming the communication landscape, necessitating new approaches to the practice and research of digital reputation management (Etter et al., 2019).

In an always-on environment, organisations of all kinds need to navigate the multitudinous possibilities - from unprecedented connectivity, engagement and 
collaboration to heightened scrutiny, criticism and falsehoods, to name a few - that can influence brand perceptions in the public sphere. Particularly since the General Data Protection Regulation (GDPR) came into effect, awareness around personal data and privacy has increased. While the responsibilities of healthcare organisations to their patients has changed significantly, there are stronger consequences for breaches of privacy. The reputation impact of privacy challenges is far-reaching affecting brand reputation, financial performance and trust (Davis, 2019). Even the supposedly "mundane" efforts to engage stakeholders on social media could pose reputation risks for healthcare organisations given the "medico-legal" ramifications (e.g. Mishra, 2019, p. 505).

Healthcare organisations find themselves at a critical juncture. With the growing use of online and social media to make healthcare decisions, providers face challenges to their reputation (in case of bad reviews) and organisational sustainability (with a potential loss of patients). However, these very developments also promise benefits for those that take the lead in embracing changes and that ensure they are ahead of the curve. The healthcare sector needs to take on board the idea that "companies that can provide greater value to consumers will benefit from increased customer satisfaction" (Cordina and Greenberg, 2020).

How, then, might communication professionals understand and manage social media use?

\section{Method}

For this study, semi-structured in-depth interviews were conducted with a purposive sample of communication professionals employed at Dutch hospitals and/or working as communication consultants for these organisations, advising them on social media strategy (e.g. Bailey, 2007; Tracy, 2013). Participating hospitals were present on several social media platforms (Facebook, Twitter or Instagram and/or YouTube) and included a link to such platforms on their official website. Interviews were conducted with 15 professionals holding such positions as "Head of Marketing and Communication" or "Advisor Online Communication" and/or "Content Manager". Interviews lasted 60-90 min and yielded a total of 326 pages of single-spaced data.

All interviews followed a semi-structured interview guide which included questions related to the importance, relevance and use of social media; the perceived opportunities and challenges of social media; and strategy to manage these affordances. Per interviewee preference and comfort, all interviews were conducted in Dutch (one of the authors is a native Dutch speaker) and transcribed verbatim, after which they were translated to English. All participants signed informed consent forms. Each interview transcript was then subjected to thematic analysis following the steps outlined by Braun and Clarke (2006): familiarisation with data; generating initial codes; searching for themes; reviewing potential themes; defining and naming themes; and producing this paper. The assessment of these themes resulted in our model articulated further as "CARE". The concerns, opportunities and interests of the interviewees became the basis for producing this model as well as a framework to re-evaluate other quotes from our sample against.

In total, data for the study included interviews with 15 participants across eight hospitals including one local hospital (Groene Hart Ziekenhuis), three specialised hospitals (Elisabeth Tweesteden, Albert Schweitzer, Maxima Medisch Centrum) and four academic hospitals (Erasmus MC, UMC Utrecht, Radboud UMC, VU MC Amsterdam). Academic hospitals are commonly identified by the abbreviation of "UMC" (University Medical Centre or Universitair Medisch Centrum) (see Table 1). 


\begin{tabular}{|c|c|c|c|c|c|}
\hline 25,2 & Participant & Department/Role & Hospital/ Organisation & $\begin{array}{l}\text { Hospital } \\
\text { type }\end{array}$ & Location \\
\hline \multirow[b]{3}{*}{130} & 1 & Marketing and Communication & $\begin{array}{l}\text { Erasmus MC and Sophia } \\
\text { Children's Hospital }\end{array}$ & Academic & Rotterdam \\
\hline & 2 & Social Media, Finance and Staff & Erasmus MC & Academic & Rotterdam \\
\hline & \multicolumn{3}{|c|}{ Corporate Website } & Academic & Rotterdam \\
\hline & 4 & Brand Manager & Erasmus MC & Academic & Rotterdam \\
\hline & 5 & $\begin{array}{l}\text { Online Marketing and } \\
\text { Communication }\end{array}$ & Het Groene Hart Ziekenhuis & Local & Gouda \\
\hline & 6 & $\begin{array}{l}\text { Online Marketing and } \\
\text { Communication }\end{array}$ & Het Groene Hart Ziekenhuis & Local & Gouda \\
\hline & 7 & Online Marketing & $\begin{array}{l}\text { UMC Utrecht and Wilhelmina } \\
\text { Children's Hospital }\end{array}$ & Academic & Utrecht \\
\hline & 8 & Communication & Maxima Medisch Centrum & Specialised & Eindhoven \\
\hline & 9 & Communications Department & Radboud UMC & Academic & Nijmegen \\
\hline & 10 & Communication & Albert Schweitzer Ziekenhuis & Specialised & Dordrecht \\
\hline & 11 & $\begin{array}{l}\text { Communication and Media } \\
\text { Relations }\end{array}$ & Albert Schweitzer Ziekenhuis & Specialised & Dordrecht \\
\hline & 12 & Online Communication & VUmc Amsterdam & Academic & Amsterdam \\
\hline & 13 & $\begin{array}{l}\text { Online Communication; Media } \\
\text { Relations }\end{array}$ & $\begin{array}{l}\text { Elisabeth- TweeSteden } \\
\text { Ziekenhuis (ETZs) }\end{array}$ & Specialised & Tilburg \\
\hline & 14 & Social Media & The Marketing Factory & & Breda \\
\hline $\begin{array}{l}\text { Table 1. } \\
\text { Participant profile }\end{array}$ & 15 & $\begin{array}{l}\text { Communication/Management } \\
\text { Consulting }\end{array}$ & Regio+ & & Rotterdam \\
\hline
\end{tabular}

\section{Results}

Drawing on interviewee perspectives, our study offers a multidimensional perspective on social media use that juxtaposes benefits and risks, opportunities and challenges. We argue that instead of viewing these social-mediated affordances as dichotomous, scholars and practitioners need to understand and manage the tensions between the risks and benefits. Deriving from our study, we conceptualise the CARE model of social media use where each dimension $-C$ (control), $A$ (accessibility), $R$ (responsiveness) and $E$ (engagement) - is unpacked through the lens of paradoxes which permits an appreciation of both/and positions instead of the polarisation implicit in either/or approaches (e.g. Poole and van de Ven, 1989). Equally, how communication professionals balance and manage these tensions will shape (variable) organisational outcomes.

Figure 1.

A visual model of CARE

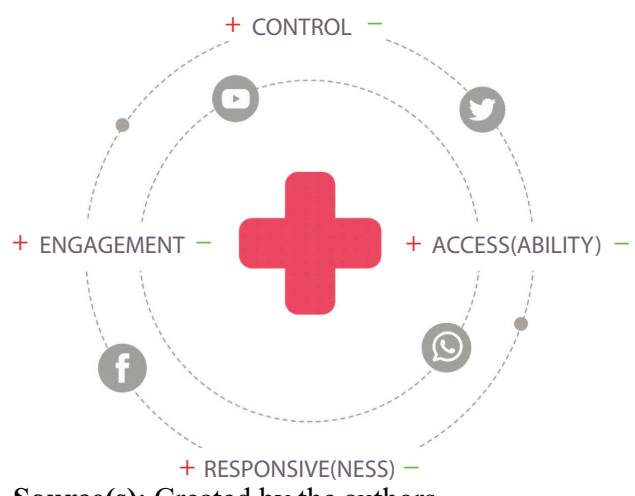

Source(s): Created by the authors 
Control: The presumed loss of control resulting from social media use has been a centre point in much corporate communication research resting on the widely used model of transmission/one-way communication whereby organisational messages could be "decoded as intended" by passive receivers ultimately allowing organisations to shape stakeholder perceptions aka reputation (e.g. Argenti, 2006). Social media, and digital technology more broadly, have allegedly disrupted such control leading some to contend that organisational control was always an elusive idea and, if anything, brands now need to cede control to gain resonance with their audiences (Fournier and Avery, 2011).

Our interviewees offered more nuanced explanations of control noting that social media in fact allowed them to exercise control by circumventing media gatekeepers and directly reaching audiences, assessing the impact of their activities and tailoring communication efforts based on stakeholder reactions. Previously done through newsletters or print magazines, current digital formats allow hospitals not only a wider reach at low cost but also bypass media gatekeepers inverting the traditional "push" relationship and facilitating an interdependent one in which organisations are "able to influence the press through channels such as Twitter or Facebook [to publish certain stories]" (P15). Moreover, the ability to "easily check the sentiment on social media" is deemed important for ensuring that communication is relevant and useful, "so you quickly get insights into what's going on ... [you can] address things well by providing particular information" (P7).

Likewise, interviewees viewed the potential to exercise control on internal stakeholders (aka employees) by instituting social media policies and guidelines to indicate "what affordances of social media are appropriate in the workplace" (Vaast and Kaganer, 2013, p. 81). Encouraging employees to speak for and on behalf of the organisation, however, highlights the paradox of employees as reputation agents, from being "untapped resources" whose creative expression can be strategically harnessed or "ticking time bombs" that pose significant reputation risk (Miles and Mangold, 2014, p. 401). The majority of participants attempt to navigate this double bind with formal policies noting that while employees are the "biggest brand ambassadors", it is too risky to not have a social media policy "to fall back on" (P12).

Nonetheless, too much control could be problematic, as it "may result in inauthentic brand communication and lead to a sense of alienation and resistance among employees" (Rokka et al., 2014, p. 807). In line with this understanding, a small minority decried the overreliance on policies as a form of control, arguing that the unpredictability of social media requires quick thinking and rigid policies and protocols might make an organisation less responsive in a crisis: “. . . but so many things happen here. So nine out of ten times you cannot even imagine them. On the spot you will have to think of how to respond and manage it, who to contact" (P10).

Instead, these participants advocate for building trust in employees to do the right thing using their best judgement. The tension between control and autonomy was aptly summarised: "you cannot control it all, you can only educate them a little and hope everyone uses their common sense" (P13) (see Table 2).

Access(ability): Interviewees view social media as affording a multi-directional communication flow between the hospital and the patient, permitting greater accessibility to the hospital and its services as well as facilitating knowledge exchange and conversation among other people, across horizontal networks. From this perspective, the patient (as consumer) feels that the hospital is more easily approachable on social media, which was regarded by interviewees as a clear advantage of having a social media presence.

Furthermore, it is easier to discuss medical topics considered more "complex" or taboo. An interviewee illustrated the case of special sessions/consultations with women regarding (pre-) menstrual symptoms or complaints organised by her hospital. Few women showed up to these sessions. Then the hospital did a session on Twitter with much success:
Digital
reputation in the healthcare sector

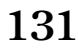


$\mathrm{JCOM}$ 25,2

\section{2}

Table 2.

Control

\section{Dimension: Control}

Positive: Perceived control over communication

(outcomes)
+ Bypassing media gatekeepers

tBpassing media gatekeepors

+ Assessing the impact of communication efforts

+ Instituting governance mechanisms such as internal training and policies

Negative: Perceived lack of control on reputation
- Challenges in employee alignment with organisational goals

- Multiplicity of actors that are involved

- Limitations of governance mechanisms such as social media policies
Illustrative quotes

[the press] will not pick up everything [published by the hospital]. You'll have to be lucky that they [the press] are just as interested in what you are doing as you are (P15)

If you place an advertisement in such a newspaper [patient magazine or local newspaper] it is difficult to see what the effect was. While if you do this online [publish an advertisement or story] you can easily measure that effect (P14) I think it would be a risk if you did not have a social media policy because you have nothing to fall back on and mostly so you know what you are doing [...] and definitely for a serious organisation like a hospital (P12)

Coincidently we have had to deal with employees grumbling about the organisation on Twitter a few times now (P9)

Yeah, but it is difficult to get 6,500 people pulling in the same direction (P13)

If you set out too many rules for everything that could go wrong, people will stop thinking on their own and they will only follow these rules (P11)

People said 'I did not dare to ask my question in public. . . but behind a computer screen people felt more safe and free, but in such a room [physical space] people did not want to talk about their (pre) menstrual symptoms or complaints but online the women did dare to ask their questions. (P14)

Stemming from the commonly invoked argument to "be where the stakeholders are", interviewees also envision long-term benefits by cultivating relationships with younger audiences. Two participants reported using social media to reach children or adolescents, for example, by experimenting with Snapchat, a social network popular among teens and millennials. Participants working at Academic Medical Centres emphasised their interest in reaching adolescents as a priority group that would constitute future medical students. In all, social media are seen as facilitators for new stakeholder relationships.

Like control, access comes with its challenges, notably the privacy associated with social media use. Legally, medical professionals are bound by rules of professional confidentiality and non-disclosure. However, reviews on the rating platform, Zorgkaart Nederland (2018) mentioned previously, reflect growing concerns with lack of privacy from sharing a room and/or rooms that cannot be locked to the lack of confidentiality in patient information in pharmacies (e.g. indiscreetly discussing medication and treatment in the presence of other patients in waiting). Besides, new complications of digital and mobile technologies additionally complicate existing offline problems.

The exponential growth of "social media photography" and short (YouTube) videos (Miller et al., 2016), not to mention the popularity of selfies, was mentioned as a specific threat to patient privacy. At one hospital, for instance, visitors and patients who make a video without respecting the privacy of other patients can be addressed by hospital security, but 
with the ease of using a mobile phone to film this has become increasingly more difficult to control. Another interviewee explained that although they do not prohibit taking selfies, they urge mindfulness: "please pay attention to the following rules: privacy of others, privacy of employees and do not disrupt your medical treatment" (P11) (see Table 3).

Responsive(ness): Relative to time invested in emails or phone calls, interviewees reported a growing use of social media channels both by patients (to ask questions of the hospital's staff) and by the hospital (to communicate with patients). Being responsive was not limited to positive sentiment; in fact, responding to negative messages or complaints received through social media was also perceived as a latent opportunity to engage with the online community even more by invoking them in resolving problems: “. . .And [uh] it will help you with your reputation especially if you can respond back with "many thanks for your tip, you were right and we are now working on improving this'... fantastic opportunity” (P15). Thus, identifying and potentially addressing brewing issues or "paracrises" (Coombs and Holladay, 2012) is critical; left unaddressed, paracrises could escalate into full-fledged crises. A case in point is the Coronavirus crisis that went far beyond such a paracrisis and required healthcare professionals to significantly increase their public healthcare responsiveness through social media and other channels. While data for this study preceded this pandemic, more research into effective responsiveness of healthcare institutions (as well as the other elements of the CARE model) for crisis situations is needed.

Owing to the fast pace of change and dual professional responsibilities (only a third of the interviewees worked fulltime on social media), participants found themselves struggling to stay updated. Equally, expectations for a speedy response are often misplaced as several intermediate experts might have to be consulted especially when communicating medical content. Particularly for the academic hospitals, where research, education and patient care are three main pillars, it can be a race against time as "social media forces you to work quicker" (P6). How to respond is also context-dependent and adds to pressure of carefully thinking through the response so as to not aggravate the issue:

\begin{tabular}{|c|c|c|}
\hline \multicolumn{2}{|c|}{ Dimension: Access(ability) } & \multirow{2}{*}{$\begin{array}{l}\text { Illustrative quotes } \\
\text { You can exchange knowledge in an } \\
\text { easily accessible manner and also } \\
\text { engage in conversation with people (P7) }\end{array}$} \\
\hline $\begin{array}{l}\text { Positive: Increased } \\
\text { access to information } \\
\text { and audience }\end{array}$ & + To information; to target audience(s) & \\
\hline & $\begin{array}{l}\text { + Access(ability) in terms of message/ } \\
\text { content comprehension }\end{array}$ & $\begin{array}{l}\text { Subjects that are often somewhat more } \\
\text { complex can be shown on a more } \\
\text { accessible level (P12) }\end{array}$ \\
\hline & + Access to future generations & $\begin{array}{l}\text { To make the younger community a } \\
\text { little more aware of what is means to be } \\
\text { healthy. So it has a more preventative } \\
\text { aim. ... to make them aware that it is } \\
\text { good to stay fit and eat healthy }(\mathrm{P} 1, \mathrm{P} 7)\end{array}$ \\
\hline $\begin{array}{l}\text { Negative: Potential to } \\
\text { cross boundaries }\end{array}$ & $\begin{array}{l}\text { - Invasion of privacy and possible } \\
\text { misuse by asking/posting sensitive } \\
\text { information/videos/photos (e.g. the } \\
\text { "selfie" culture) }\end{array}$ & $\begin{array}{l}\text {. . you cannot just participate in the } \\
\text { conversation on social [media] because } \\
\text { there is a lot of personal information. } \\
\text { Yes, you are dealing with a disease of a } \\
\text { patient. The patient does not want } \\
\text { everything out in the open, but you do } \\
\text { want to be empathetic and not too } \\
\text { professional and bleak. So you'll have } \\
\text { to find a middle ground (P2, P3) }\end{array}$ \\
\hline
\end{tabular}

Digital reputation in the healthcare sector

133
Table 3. Access(ability) 
$\mathrm{JCOM}$

25,2

134

It is all really customised. So, it is important to not always react impulsively, yes think for more than 3 seconds. ... but you have to realise the consequences of that message, you know. And what sort of language you will use and how you would interpret that sentence. (P13)

In addition to the issue of feasibility is the question of prioritising. The growing use of social media in their organisations prompted some to introspect on the core purpose of the healthcare institution. For example, an interviewee noted that reputation problems stemming from negligence in patient care and well-being would have more serious implications than what an organisation does (not do) on social media:

A few months ago, we had an IVF-disaster, which is way worse for your image, because it resulted in a very big group of patients who became victims. They did not know whether they would have a child from the man they thought they would. Yes, that is a lot worse. That is directly related to care giving. (P7).

Others concurred but shared examples consistent with the amplifying effect of social media as an "accessory in escalating crises" (Mei et al., 2010, p. 143) such as the time a patient posted a picture on Twitter:

Like a lot of dust on the light cover [above the patient's head when they woke up from surgery]. So that patient took a picture of that and that was really horrible of course. This escalated on social media ... that really was reputation damage for the organisation. ... Yes 'is it a clean place?' that is essential for a hospital, hygiene. So that is then a reputation thing. (P2) (see Table 4).

Engagement: The final dimension in our model refers to engagement, arguably, the holy grail of social media practice and scholarship. The primacy of engagement via interactive, dialogic affordances of social media is well documented; in practice, however, much organisational (corporate and non-profit) research confirms the preponderance of broadcasting/information dissemination approaches, as opposed to engagement (e.g. Etter, 2014; Lovejoy et al., 2012).

For our interviewees, engagement manifested in the ability to position the hospital as a caring institution, to "convey a warm impression. . .to show the human dimension of care"

Dimension: Responsive(ness)
$\begin{aligned} & \text { Positive: Opportunity to }+ \text { Ability to respond quickly } \\ & \text { respond quickly }\end{aligned}$
respond quickly

Negative: Alignment with core purpose may diverge
- Creates an always-on environment

- "Forced innovation" that may contradict core purpose (providing quality healthcare)
Illustrative quotes

I notice it myself that if I need to talk to an organisation I use social media because if I email you often get an email back with "we will answer within two weeks." So I thought I'd try social media, and then you get an answer almost immediately, which is really nice. Yes, and I think that happens here too. We try to answer as quickly as possible (P9) Social media forces you to work quicker (P6)

I do not think hospitals are particular organisations that are on the frontline when it comes to innovation, well with medical innovation they are of course. But when it comes to communication and social media they are not necessarily, and we always are a little behind the curve even if you have a different target group, you still need to keep renewing. We do not want to be doing the same thing we did two years ago and want to keep things exciting (P12)
Table 4.

Responsive(ness) 
(P6, P11) with a goal to “... .eventually to have engagement on your brand” (P4). Participants explained that via social-mediated communication, massive organisations such as hospitals can be humanised and made less impersonal. The underlying driver, according to some, was that its institutional status made hospitals more distant, thereby limiting the connectedness with their audience. Social media were seen as a way to communicate the faces behind the hospital and alleviate perceptions of "a cumbersome organisation where all doors are closed and one you cannot come to easily" (P9).

Participants relayed the importance of storytelling as the pathway to dialogue and conversation. Another shared her vision for storytelling as facilitating dialogue:

Well I would like to tell more stories and we will post that on all our social channels and then try to generate dialogue with our target group. So it is not only sending out messages, but also entering into discussions with our target groups. (P4).

Another narrated a poignant story about her hospital facilitating the journey of a terminally ill patient to visit his mother's grave in Morocco. Not only was the effort well received on social media but many employees expressed pride in their employer.

Coupled with greater engagement via dialogue comes the possibility of negative feedback, making it imperative for communication or social media professionals to have the know-how and guidance to respond in an appropriate manner to questions and complaints posted on social media. None of the participants in this study were authorised to answer medical questions posed on social media. Additionally, social media professionals were not authorised to (or able to - as they have no access to a patient's medical file) correct, dispute or provide nuance to, inaccurate messages posted by disgruntled patients or their family members.

A broader concern relates to the consequences of anonymity afforded by social-mediated engagement. On the one hand, social media are seen as empowering previously powerless groups or individuals; on the other, the anonymity affords them the possibility to distort information and spread falsehoods that bear serious reputation consequence. While acknowledging the need to understand the "sentiment behind the message", interviewees highlighted unequal power relationship that disadvantages the institution because "people always believe the patient" (P8). Combined with the inability to "defend" the brand, our interviewees highlight the unequal power equation that they are faced with on social media, particularly as engagement with a somewhat anonymous public is difficult to sustain.

However, practitioners may take the opportunity not to engage. While they should know the risks of not mitigating negative comments, some practitioners are and more should be aware that these engagements on social media are fleeting and transient but simultaneously timeless and preserved. This struggle relates to a recognition that what is important at present may not be on the agenda tomorrow (see Table 5).

\section{Implications and discussion}

This exploratory study examined how healthcare organisations perceive the (reputational) opportunities and challenges of social media for their praxis. Participant perspectives shed light on how hospitals must simultaneously navigate the use of social media from a strategic perspective. Based on our participants' perspectives, we advance the CARE model, highlighting four tensions that communication professionals must balance and manage in order to be able to shape (variable) organisational outcomes.

Currently, there are no models of social-mediated communication in peer-reviewed outlets that respond to this challenge. Two models that are tangentially related are the SPIN model (Mills, 2012) and Technology Acceptance Model (TAM) (Davis, 2019), which has been adjusted to fit the assessment of social media adoption. The SPIN model focusses on the how to create viral content and does not provide insight into the challenges that social media 
$\mathrm{JCOM}$

Negative: Managing negative feedback constructively 25,2

Dimension: Engagement

Positive: Shaping the connection with the audience

\section{6} + Humanising the brand through
storytelling and dialogue

+ Fleeting
- Lack of knowledge/resources/ decision-making power

- Challenge in correcting mis(perceptions); negative criticism/feedback

- Unequal power equation
Table 5.

Engagement
Illustrative quotes

The reason for profiling, so you are more visible as an organisation is to attract patients (P11)

it [social media] is fleeting, so on that day and maybe the next two days it might be hot topic. And afterwards it might still simmer a little, but if there is something positive the next day, you'll be viewed positively in the news. That is something to keep in mind: that [social media] are fleeting, tomorrow people might all forget it again, as it were (P13)

I even find it a challenge to just point people in the right direction.... I always feel responsible when I get those sort of messages, and think "oh no, it is my job to ensure this person will hear something from us" (P9)

People always believe the patient (P8)

... you can just hurl a lot of things very easily into the world. I cannot do anything about that, but I do wonder if that is a good thing? All those "klokkenluiders" [someone who publishes something that might damage the reputation of the organisation] (...) sometimes you're happy with them as it can bring about improvement. On the other hand, the sentiment that is conveyed is rather simplistic (P7)

experts are facing. TAM and its variations (see e.g. Raunier et al., 2014) tend to focus more on the acceptance and use of new technology, but do not address actual or successful use of social media (by individuals). This approach also has limited applications to assess the challenges communication professionals face on social media, both in general and in healthcare organisations.

The CARE model has developed with a focus on actual practices by practitioners and moves away from such understandings of social-mediated communication that tend towards either/or choices or acceptance levels. Our model instead underlines how social media both increase and decrease the tensions for the dimensions control, access(ability), responsive(ness) and engagement. In doing so, the CARE model provides a comprehensive perspective of social media use by healthcare organisations and its practical implications for reputation management. Our model is unique in the sense that currently no models have been found in peer-reviewed journals that describe social-mediated communication and involve the associated practical challenges that social media professionals face.

We believe our results can offer an initial framework for interpretation and analysis in other areas but have critical application in the healthcare sector. As explicated in the results section, each dimension of the CARE model presents organisations with competing tensions that need to be simultaneously navigated. Particularly for two of the four dimensions, that is, Control and Engagement, the results challenge the polarised positions (negative and positive 
respectively) which are accorded to these dimensions in most scholarship. As explicated before, participant experiences suggest that the perceived loss of control over "official" messaging co-exists with a sense of control that comes from circumventing media gatekeepers and getting the organisational voice out. Similarly, the potential to institute guidelines for employee behaviour provides a semblance of control over the multiplicity of voices that may speak on behalf of the organisation. However, the mere presence of a social media policy does not make an organisation immune to potential risks. O'Connor et al. (2016) concur that for policies to be effective, they have to be enforced and embedded in the organisation through appropriate employee communication and training.

Likewise, humanising a hospital or healthcare organisation presents trade-offs in terms of knowhow, expertise and decision-making abilities to effectively engage or respond to the challenges of engagement. The promise of access(ability) - of and to information, audiences and new stakeholder groups - demands finding a middle ground, given that some of the topics regarding specific medical conditions are personal and private to the patient. This makes it difficult to respond to patients who share their story online and safeguard their privacy. Finally, organisational responsive(ness) is deemed critical in maintaining (online) relations particularly in the face of a negative situation such as consumer complaints (e.g. Einwiller and Steinel, 2015). Although responsiveness can enhance positive perceptions of an organisation, healthcare professionals in our study noted the challenge of not only being behind the innovation curve but also questioning whether social media fit with the goals of delivering quality healthcare. Collectively, the model affirms the paradoxical manifestations of CARE dimensions and validates the need to foreground field experiences in social media research.

Additionally, the model offers some big picture reflections with implications for reputation in the health sector. As stakeholders increasingly expect transparent healthcare information (e.g. Cordina and Greenberg, 2020), the power equation shifts in favour of the patient, who, as we have found, now holds a better informed and empowered position compared to the past. However, when voicing patient complaints online, especially when these are false, they "force organisations to respond to the beliefs or new expectations that it propagates" (Aula and Heinonen, 2016, p. 145). This in turn can lead to a mismatch or misunderstanding of expectations and opinions. Because organisations "are easily stigmatized as manipulators" (Aula and Heinonen, 2016, p. 148), it can be difficult to respond to negativity on social media. Therefore, by monitoring social media conversations about the organisation, negative sentiment or possible reputation risk can be intercepted.

For healthcare organisations, ethical issues of privacy and anonymity constitute a particularly serious challenge to establishing and maintaining trustworthy relationships between healthcare providers and patients. The conundrum between being transparent and compliant means that even storytelling needs to be undertaken within the norms of professional medical practice without compromising patient privacy or confidentiality. Conversations about privacy and anonymity also raise the question of "power" and control whereby patients are able to share (personal, positive and/or negative) information online but hospitals and their social media representatives are limited by rules and regulations. This creates an unequal playing field, limiting hospitals' ability to respond well and provide more context to a situation that may have been presented unfairly, thereby amplifying the reputation risk.

Such an imbalance is exacerbated due to the privacy-sensitive nature of health data (in context of the GDPR). In light of a participant's comment that "people always believe the patient", we find a hint of a dynamic that resembles of the underdog against the big corporation (Zourrig and El Hedhli, 2018). Such an imbalanced power equation may apply to a wider range of organisations and sectors, and so the CARE model is likely to have useful applications beyond healthcare.

An interesting finding, worthy of further exploration, relates to the potential of branding hospitals and healthcare organisations on social media. At one level, this approach is attuned 
$\mathrm{JCOM}$

25,2

to the previously discussed shifts towards a patient-as-consumer profile and a competitive healthcare landscape characterised by heightened (media) scrutiny of medical practice and demand for transparency. However, it is equally illustrative of the everyday communication practices in which hospitals engage in "doing branding" (Vasquez et al., 2013). By attending to the communication dynamics, we can appreciate the negotiated and diffuse processes of branding in which, for better or worse, patients, employees and the organisation are all able to shape and define the brand.

Related to branding is another paradox - on the one hand, healthcare organisations are (seen as) brands competing for attention and "customers"; on the other, their primary goal is to deliver quality healthcare. In characterising their organisations as "brands", participants reflect a keen awareness of business developments in the healthcare sector, that is, greater competition and more choice for the consumer. However, this demonstrates the challenges previously outlined in how such brand can engage the wider public on social media as participants who were "uninvited" (Fournier and Avery, 2011). Part of this is to focus on connected online communities often referred to as "brand communities". These may positively contribute to enhancing brand trust and loyalty (Laroche et al., 2012). Given that brand loyalty closely relates to attitudinal perspectives of consumers (Zheng et al., 2015), it is also relevant to an organisation's overall reputation. Arguably, branding and healthcare provision are no longer mutually exclusive, especially in the digital age. Still, more research is necessary to ascertain the reputation damage in case of lapses in caregiving relative to brand challenges in the social media domain.

The CARE model is based on qualitative, exploratory research using a small data set, which presents several limitations in terms of generalisability and validity. Although the use of qualitative methods permits a foregrounding of participant perspectives, a larger data set as well as the implementation of some quantitative methods would allow for more generalisation and permit testability. The model itself may also vary in applicability across different healthcare contexts. For example, the applicability in places where patients cannot choose their healthcare provider limits the generalisability of this Dutch experience. Future research may examine whether and how the dimensions of CARE manifest in other contexts.

Despite these limitations, the CARE model provides a valuable contribution for media and communication scholars in search of a deeper understanding of the impacts of socialmediated communication in the healthcare sector. Our focus on paradoxes allows us to move away from the either/or and instead adopt a perspective of both/and. This results in a more insightful and sophisticated view on what is considered a ubiquitous phenomenon, and in doing so, we establish the significance of the CARE model. Future research may examine the applicability of this model to other organisational domains. The issues highlighted in this study will no doubt take on greater import in the post Coronavirus era, as public health will remain a crucial issue more broadly, and healthcare organisations will continue to work through the paradoxical problems and possibilities outlined in our CARE model. While our research preceded the COVID-19 pandemic, our model begins to highlight the interplay of organisational identity and culture in defining the possibilities for social media use.

Few studies have investigated the role of culture in shaping social media use in organisations (e.g. see Rokka et al., 2014), and examples from industry are scattered between open, collaborative cultures that encourage participation to those at the other end of the continuum. The culture of openness no doubt comes with its own paradox as it can often be counter-intuitive to organisational interests (Macnamara and Zerfass, 2012), manifested, for example, in the tension between controlling and encouraging employee voice on social media. Being aware of these tensions is indeed critical to identifying a strategic approach that works best for each organisation. In foregrounding these tensions, we hope have shown that the CARE model offers a grounded approach, situated in the practical realities and experiences of professionals and goes beyond the presumed dichotomies of risks and benefits. 


\section{References}

Argenti, P.A. (2006), "How technology has influenced the field of corporate communication", Journal of Business and Technical Communication, Vol. 20 No. 3, pp. 357-370, doi: 10.1177/ 1050651906287260.

Aula, P. and Heinonen, J. (2016), The Reputable Firm: How Digitalization of Communication Is Revolutionizing Reputation Management, Springer International.

Bailey, C. (2007), A Guide to Qualitative Field Research, 2nd ed., Sage Publications, California.

BBC (2014), "Social media fuel rise in complaints against doctors", 21 July, available at: https://www. bbc.co.uk/news/health-28368216 (accessed 4 June 2019).

Bermúdez-Tamayo, C., Alba-Ruiz, R., Jiménez-Pernett, J., Gracia Gutiérrez, J.F., Travel- Salcedo, V. and Yubraham-Sánchez, D. (2013), "Use of social media by Spanish hospitals: perceptions, difficulties, and success factors", Telemedicine and e-Health, Vol. 19 No. 2, pp. 137-145.

Braun, V. and Clarke, V. (2006), "Using thematic analysis in psychology", Qualitative Research in Psychology, Vol. 3 No. 2, pp. 77-101.

CBS (2013), "Woman sues Northwestern after doctor posted drunk photos", 21 August, available at: https://chicago.cbslocal.com/2013/08/21/woman-sues-northwestern-after-doctor-posted-drunkphotos/ (accessed 16 May 2019).

Coombs, W.T. and Holladay, S.J. (2012), "The paracrisis: the challenges created by publicly managing crisis prevention”, Public Relations Review, Vol. 38 No. 3, pp. 408-415, doi: 10.1016/j.pubrev.2012. 04.004 .

Cordina, J. and Greenberg, S. (2020), "Consumer decision making in healthcare: the role of information transparency", McKinsey Quarterly, available at: https://www.mckinsey.com/industries/ healthcare-systems-and-services/our-insights/consumer-decision-making-in-healthcare-the-roleof-information-transparency.

Davis, M. (2019), “4 damaging after-effects of a data breach", available at: https://www. cybintsolutions.com/4-damaging-after-effects-of-a-data-breach/ (accessed 22 August2020).

de Koning, A. (2017), "Ongeluk Vader Roept Vragen Op”, AD Rotterdam, available at: https://www.ad.n1/ rotterdam/ongeluk-vader-in-centrum-rotterdam-roept-vragenop a52ebb8b/(accessed 28 January).

Deloitte (2019), "Global health care outlook: shaping the future", available at: https:/www2.deloitte. com/content/dam/Deloitte/global/Images/infographics/lifesciences-healthcare/gx-lshc-hcoutlook-2019-infographic.pdf (accessed 4 June 2019).

Einwiller, S.A. and Steinel, S. (2015), "Handling complaints on social network sites - an analysis of complaints and complaint responses on Facebook and Twitter pages of large US companies", Public Relations Review, Vol. 41 No. 2, pp. 195-204, doi: 10.1016/j.pubrev.2014.11.012.

Etter, R., Ravasi, D. and Elaenor, C. (2019), "Social media and the formation of organizational reputation", Academy of Management Review, Vol. 44 No. 1, pp. 28-52, doi: 10.5465/amr.2014.0280.

Etter, M. (2014), "Broadcasting, reacting, engaging - three strategies for CSR communication in Twitter", Journal of Communication Management, Vol. 18 No. 4, pp. 322-342, doi: 10.1108/JCOM01-2013-0007.

European Communication Monitor (2019), available at: http:/www.communicationmonitor.eu/wpcontent/uploads/dlm_uploads/ECM19-European-Communication-Monitor-2019.pdf.

Fombrun, C. and van Riel, C. (2007), Essentials of Corporate Communication Implementing Practice for Effective Reputation Management, Routledge, London.

Fombrun, C. (1996), Reputations, Harvard Business School Press, Boston, MA.

Fournier, S. and Avery, J. (2011), “The uninvited brand”, Business Horizons, Vol. 54 No. 3, pp. 193-207.

Jiang, S. (2019), "Functional interactivity in social media: an examination of Chinese health care organizations' microblog profiles”, Health Promotion International, Vol. 34 No. 1, pp. 38-46, doi: 10.1093/heapro/dax056.

reputation in the healthcare sector

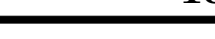


$\mathrm{JCOM}$ 25,2
Kaplan, A.M. and Haenlein, M. (2010), "Users of the world, unite! the challenges and opportunities of social media”, Business Horizons, Vol. 53 No. 1, pp. 59-68.

Keller, B., Labrique, A., Jain, K.M., Pekosz, A. and Levine, O. (2014), "Mind the gap: social media engagement by public health researchers", Journal of Medical Internet Research, Vol. 16 No. 1, [Online publication], doi: 10.2196/jmir.2982.

Kotsenas, A.L., Arce, M., Aase, L., Timimi, F.K., Young, C. and Wald, J.T. (2018), "The strategic imperative for the use of social media in health care", Journal of American College of Radiology, Vol. 15, pp. 155-161, doi: 10.1016/j.jacr.2017.09.027.

Laroche, M., Habibi, M., Richard, M. and Sankaranarayanan, R. (2012), "The effects of social media based brand communities on brand community markers, value creation practices, brand trust and brand loyalty", Computers in Human Behavior, Vol. 28 No. 5, pp. 1755-1767.

Lim, W.M. (2016), "Social media in medical and health care: opportunities and challenges", Marketing Intelligence \& Planning, Vol. 34 No. 7, pp. 964-976, doi: 10.1108/MIP-06-2015-0120.

Lovejoy, K., Waters, R.D. and Saxton, G.D. (2012), "Engaging stakeholders through Twitter: how nonprofit organizations are getting more out of 140 characters or less", Public Relations Review, Vol. 38 No. 2, pp. 313-318.

Macnamara, J. and Zerfass, A. (2012), "Social media communication in organizations: the challenges of balancing openness, strategy, and management", International Journal of Strategic Communication, Vol. 6, pp. 287-308, doi: 10.1080/1553118X.2012.711402.

McCarroll, M.L., Armbruster, S.D., Chung, J.E., Kim, J., McKenzie, A. and von Gruenigen, V.E. (2014), "Health care and social media platforms in hospitals", Health Communication, Vol. 29 No. 9, pp. 947-952, doi: 10.1080/10410236.2013.813831.

Mei, J.S.A., Bansal, N. and Pang, A. (2010), "New media: a new medium in escalating crisis?”, Corporate Communications: An International Journal, Vol. 15 No. 2, pp. 143-155.

Miles, S.J. and Mangold, W.G. (2014), "Employee voice: untapped resource or social media time bomb?", Business Horizons, Vol. 57, pp. 401-11, doi: 10.1016/j.bushor.2013.12.011.

Miller, D., Costa, E., Haynes, N., McDonald, T., Nicolescu, R., Sinanan, J., Spyer, J., Venkatraman, S. and Wang, X. (2016), How the World Changed Social Media, UCL Press, University College London.

Mills, A.J. (2012), "Virality in social media: the SPIN framework", Journal of Public Affairs, Vol. 12 No. 2, pp. 162-169, doi: 10.1002/pa.1418.

Mishra, S. (2019), "When patients connect with physicians on Facebook: physician perspectives on benefits, challenges and strategies for managing interaction", Health Technology, Vol. 9 No. 4, pp. 505-515, doi: 10.1007/s12553-018-0273-Z.

NVZ (2011), "Zorg op doorreis. Brancherapport ziekenhuizen 2011", Report of the Nederlandse Vereniging van Ziekenhuizen, available at: https://www.sirm.nl/docs/Publicaties/NVZ-2011zorg-op-doorreis.pdf.

O'Connor, K.W., Schmidt, G.B. and Drouin, M. (2016), "Helping workers understand and follow social media policies”, Business Horizons, Vol. 59 No. 2, pp. 205-211, doi: 10.1016/j.bushor.2015.11.005.

Poole, M.S. and van de Ven, A. (1989), "Using paradox to build management and organizational theories", Academy of Management Review, Vol. 14 No. 4, pp. 562-578.

Raunier, R., Rawski, G., Yang, J. and Johnson, B. (2014), "Technology acceptance model (TAM) and social media usage: an empirical study on Facebook", Journal of Enterprise Information Management, Vol. 27 No. 1, pp. 6-30, doi: 10.1108/JEIM-04-2012-0011.

Ravasi, D., Rindova, V., Etter, M. and Cornelissen, C. (2018), "The formation of organizational reputation", Academy of Management Annals, Vol. 17 No. 2, pp. 574-599, doi: 10.5465/annals. 2016.0124.

Richter, J.P., Muhlestein, D.B. and Wilks, C.E. (2014), "Social media: how hospitals use it, and opportunities for future use", Journal of Healthcare Management, Vol. 59 No. 6, pp. 447-460. 
Rokka, J., Karlsson, K. and Tienari, J. (2014), "Balancing acts: managing employees and reputation in social media”, Journal of Marketing Management, Vol. 30 Nos 7-8, pp. 802-827.

RVZ (2010), "Health 2.0: it's up to you", available at: https:/www.hon.ch/Global/pdf/CONF10322/ RVZreportHealth2.0It\%27suptoyou.pdf.

Tracy, S. (2013), Qualitative Research Methods Collecting Evidence, Crafting Analysis, Communicating Impact, Wiley-Blackwell, West Sussex.

Vaast, E. and Kaganer, E. (2013), "Social media affordances and governance in the workplace: an examination of organizational policies", Journal of Computer-Mediated Communication, Vol. 19, pp. 78-101, doi: 10.1111/jcc4.12032.

Van de Belt, T.H., Berben, S.A., Samsom, M., Engelen, L.J. and Schoonhoven, L. (2012), "Use of social media by Western European hospitals: longitudinal study", Journal of Medical Internet Research, Vol. 14 No. 3, p. e61, doi: 10.2196/jmir.1992, [Online publication].

van de Belt, T.H., Engelen, L.J., Berben, S.A., Teerenstra, S., Samsom, M. and Schoonhoven, L. (2013), "Internet and Social media for health-related information and communication in health care: preferences of the Dutch general population”, Journal of Medical Internet Research, Vol. 15 No. 2, p. e220, doi: 10.2196/jmir.2607, [Online publication].

Vasquez, C., Sergi, V. and Cordelier, B. (2013), "From being branded to doing branding: studying representation practices from a communication-centered approach", Scandinavian Journal of Management, Vol. 29 No. 2, pp. 135-146.

Ventola, C. (2014), "Social media and health care professionals: benefits, risks, and best practices", Pharmacy and Therapeutics, Vol. 39 No. 7, pp. 491-499.

Vogel, L. (2019), "Medicine slow to recognize social media as window into the patient experience", Canadian Medical Association Journal, Vol. 191 No. 3, pp. E87-E88, doi: 10.1503/cmaj.109-5701.

Vuijst, C. (2009), "Liever bekwaam dan aardig: Professor Margot Trappenburg over artsen en patiënten bejegening", Monitor, Vol. 39 No. 6, pp. 18-23.

Waeraas, A. and Maor, M. (Eds) (2015), Organisational Reputation in the Public Sector, Routledge, New York.

Zarkada, A.K. and Polydorou, C. (2014), "You might be reputable but are you 'liked'?: orchestrating corporate reputation co-creation on Facebook", Social Media in Strategic Management, Vol. 11, pp. 87-113, doi: 10.1108/S1877-6361\%282013\%290000011009.

Zheng, X., Cheung, C., Lee, M. and Liang, L. (2015), "Building brand loyalty through user engagement in online brand communities in social networking sites", Information Technology \& People, Vol. 28 No. 1, pp. 90-106, doi: 10.1108/ITP-08-2013-0144.

Zorgkaart Nederland (2018), "Privacy en zorg gaan niet goed samen", available at: https://www. zorgkaartnederland.nl/feiten-en-cijfers/privacy-en-zorg-gaan-niet-goed-samen (accessed 4 June 2019).

Zourrig, H. and El Hedhli, K. (2018), "Consumers' motivations and roles in rooting for or against underdog consumer", International Journal of Consumer Studies, Vol. 42 No. 1, pp. 164-172, doi: 10.1111/ijcs.12395.

\section{Corresponding author}

Vidhi Chaudhri can be contacted at: chaudhri@eshcc.eur.nl
Digital

reputation in

the healthcare

sector 